\title{
ENHANCEMENT OF THE BASE FOR 3,7-DISUBSTITUTED $[1,2,4]$ TRIAZOLO[4,3-a]PYRAZIN-8(7H)-ONE DERIVATIVES AS PROMISING PHARMACEUTICAL AGENTS
}

\author{
K.Yu.Kulikovska, S.S.Kovalenko, O.G.Drushlyak, I.O.Zhuravel, S.M.Kovalenko \\ National University of Pharmacy \\ 53, Pushkinska str., Kharkiv, 61002. E-mail: kulikovskaja.k@gmail.com
}

Key words: 3-hydrazinopyrazin-2(1H)-one; [1,2,4]triazolo[4,3-a]pyrazin-8(7H)-one; cyclization

A suitable and effective scheme for the synthesis of 3,7-disubstituted [1,2,4]triazolo[4,3-a]pyrazin-8(7H)-ones has been suggested and tested. It can provide a wide chemical diversity of the final products containing practically any substituent in position 3. The scheme previously developed starts from esters of oxalamic acid following with the cyclization of intermediate 3-hydrazinopyrazin-2-ones with carbonyl-containing compounds (orthoesters or alkylcarbonic acid anhydrides). To introduce aryl or heteryl substituents in position 3 of the heterocyclic system we propose to use the reaction of 3-hydrazinopyrazin-2-ones with the corresponding carbonic acids preliminary activated by carbonyldiimidazole (CDI). The further cyclization is carried out by reflux for 24 hours in anhydrous DMFA. The structure of the compounds obtained has been proven by elemental analysis and ${ }^{1} \mathrm{H}$ NMR spectroscopy data. Formation of [1,2,4]triazolo[4,3-a]pyrazin-8(7H)-one condensed system is in good correlation with spectral data, and is confirmed by the presence of signals of $\mathrm{H}-5$ and $\mathrm{H}-6$ protons of the pyrazinone fragment as doublets at $\delta$ 7.15-7.28 ppm and $\delta$ 7.50-7.59 ppm, respectively. The compounds synthesized are of particular interest as potential pharmacological objects with the cytotoxic, membrane-stabilizing, cerebroprotective, cardioprotective activity.

РОЗШИРЕННЯ БАЗИ ПОХІДНИХ 3,7-ДИЗАМІЩЕНИХ 7Н-[1,2,4]ТРИАЗОЛО[4,3-а]ПІРАЗИН-8-ОНІВ ЯК ПЕРСПЕКТИВНИХ ФАРМАЦЕВТИЧНИХ АГЕНТІВ

К.Ю.Куликовська, С.С.Коваленко, О.Г.Друшляк, І.О.Журавель, С.М.Коваленко

Ключові слова: 3-гідразинопіразин-2-он; [1,2,4]триазоло[4,3-а]піразин; циклізація

Запропоновано і апробовано зручну та ефективну схему синтезу 3,7-дизаміщених 7Н-[1,2,4]триазоло [4,3-а]піразин-8-онів, що здатна забезпечити велике хімічне різномаїття кінцевих продуктів, які можуть мати практично будь-який замісник у 3 положенні. Попередньо розроблена нами схема виходить з естерів оксаламових кислот з подальшою циклізацією проміжних 3-гідразинопіразин-2-онів з карбоніловмісними сполуками (ортоестерами і ангідридами алкілкарбонових кислот). Для введення в положення З гетероциклічної системи арильних та гетерильних замісників ми пропонуємо використовувати реакцію 3-гідразинопіразин-2-онів з відповідними карбоновими кислотами, які попередньо активували карбонілдіімідазолом (КДІ). Подальшу циклізацію здійснювали при кип'ятінні в ДМФА впродовж 24 годин. Структура отриманих сполук доведена за допомогою елементного аналізу та даних ${ }^{1} \mathrm{H}$ ЯMPспектроскопії. Утворення конденсованої системи [1,2,4]триазоло[4,3-а]піразин-8-ону добре узгоджується зі спектральними даними та підтверджується наявністю сигналів протонів Н-5 та H-6 піразинонового фррагменту, які проявляються як дублетні сигнали при б 7.15-7.28 м.ч. та $\delta$ 7.50-7.59 м.4., відповідно. Синтезовані сполуки представляють певний інтерес як потенційні фрармакологічні об'єкти з цитотоксичною, мембраностабілізуючою, церебропротекторною, кардіопротекторною активностю.

РАСШИРЕНИЕ БАЗЫ ПРОИЗВОДНЫХ 3,7-ДИЗАМЕЩЕННЫХ 7Н-[1,2,4]ТРИАЗОЛО[4,3-а]ПИРАЗИН-8ОНОВ КАК ПЕРСПЕКТИВНЫХ ФАРМАЦЕВТИЧЕСКИХ АГЕНТОВ

К.Ю.Куликовская, С.С.Коваленко, А.Г.Друшляк, И.А.Журавель, С.Н.Коваленко

Ключевые слова: 3-гидразинопиразин-2-он; [1,2,4]триазоло[4,3-а]пиразин; циклизация

Предложена и апробирована удобная и эфрфрективная схема синтеза 3,7-дизамещенных 7H-[1,2,4]mpuазоло[4,3-а]пиразин-8-онов, которая способна обеспечить большое химическое разнообразие конечных продуктов, содержащих практически любой заместитель в 3 положении. Предварительно разработанная нами схема исходит из эфриров оксаламовых кислот с последующей циклизацией промежуточных 3-гидразинопиразин-2-онов с карбонилсодержащими соединениями (ортоэфрирами и ангидридами алкилкарбоновых кислот). Для введения в положение 3 гетероциклической системы арильных и гетерильных заместителей мы предлагаем использовать реакцию 3-гидразинопиразин-2-онов с соответствующими карбоновыми кислотами, которые предварительно активировали карбонилдиимидазолом (КДИ). Дальнейшую циклизацию осуществляли при кипячении в ДМФА в течение 24 часов. Структура полученных соединений доказана с помощью элементного анализа и данных ${ }^{1} \mathrm{H}$ ЯMP-спектроскопии. Образование конденсированной системы [1,2,4]триазоло[4,3-а]пиразин-8-она хорошо согласуется со спектральными данными и подтверждается присутствием сигналов протонов H-5 ma H-6

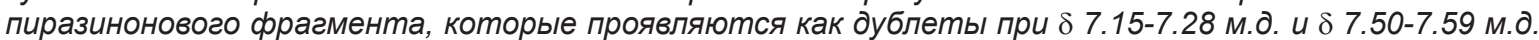
Синтезированные соединения представляют определенный интерес как потенциальные фрармакологические объекты с цитотоксичной, мембраностабилизирующей, церебропротекторной, кардиопротекторной активностью. 
Modern research in the therapy of oncological diseases testify an essential role of glutamic acid derivatives in cancer pathogenesis. Substances competitively inhibiting glutamate in the interaction with AMPA- and NMDA-receptors will be most likely very useful in oncopathology treatment $[1,2]$. Therefore, a promising area of pharmaceutical science is further study of $[1,2,4]$ triazolo[4,3-a]pyrazines, which exhibit the pharmacological effects mentioned above, as well as creation of medicines on their basis.

With the help of the computer programme PASS (Prediction of Activity Spectra for Substances) [3], which enable to estimate the pharmacological effect, the mechanism of action and specific toxicity of a substance, we carried out the investigation of the base of $N^{7}$-aryl-[1,2,4]triazolo[4,3-a]pyrazines derivatives with alkyl substituents in position 3 previously synthesized [4]. Computational results indicate a high potential of cytotoxic, membrane-stabilizing, cerebroprotective, cardioprotective activity of the compounds obtained.

Variation of substituents in molecules with the same heterocyclic structure can affect the ligand-receptor interaction; it can be relevant in therapy of not only oncopathologies, but many diseases associated with the protein-receptor competitive antagonism.

On the basis of analysis of the chemical potential of $[1,2,4]$ triazolo $[4,3-a]$ pyrazine structure, which is bioisosteric analogue of nucleic acids, the most promising randomization points of this basic structure

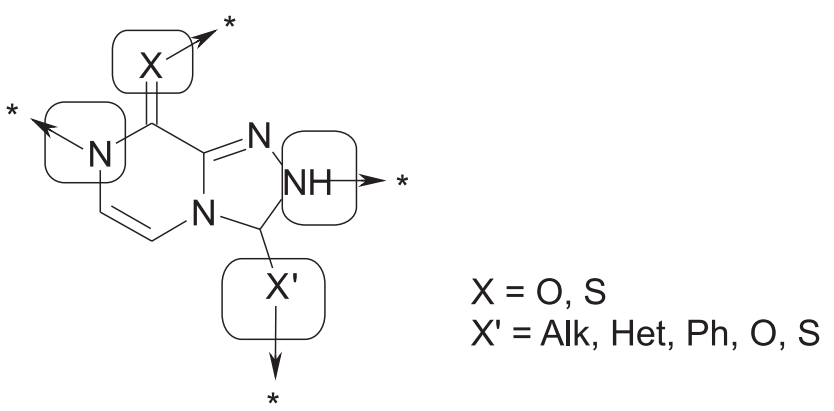

Fig. The basic structure of the focused combinatorial library based on $[1,2,4]$ triazolo[4,3-a]pyrazine. have been determined and the synthetic transformation schemes that are suitable for focused combinatorial libraries construction have been developed.

The scheme of $[1,2,4]$ triazolo $[4,3-a]$ pyrazines synthesis that is used in organic synthesis the most frequently starts from 2,3-dichloropyrazine and consists in cyclization of 2-chloro-3-hydrazinopyrazine obtained under the action of acid halides of carbonic acid with hydrolysis of intermediate 8-chloro[1,2,4] triazolo[4,3-a]pyrazine and subsequent $N$-alkylation [5]. The principal lack of this method is a limited set of substituents in position 7 of the cyclization product. In our previous work approaches to $N^{7}$-substituted $[1,2,4]$ triazolo $[4,3-a]$ pyrazines with alkyl and aryl substituents in position 7 were discussed. The aim of the present paper is the use of a wide range of carbonyl reagents for modification of position 3 in the target $[1,2,4]$ triazolo[4,3- $a]$ pyrazines by various substituents.

\section{Results and Discussion}

Earlier we reported that we had developed the scheme of the synthesis of the target $N^{7}$-aryl $[1,2,4]$ triazolo [4,3- $a$ ] pyrazines starting from esters of oxalic acid monoamides via stages of formation of $N^{1}$-arylpyrazin-2,3-diones, $N^{1}$-aryl-3-chloropyrazin-2-ones and $N^{1}$-aryl-3-hydrazinopyrazin-2-ones [4]. In further cyclization we used ortho-esters and anhydrides of carbonic acids. By this method 3-alkyl- $N^{7}$-aryl-[1,2,4] triazolo[4,3- $a]$ pyrazin-8(7H)-ones were synthesized.

The aim of our next work was enhancement of the series of $N^{7}$-substituted $[1,2,4]$ triazolo[4,3-a]pyrazin$8(7 H)$-ones previously synthesized by means of aryl or heteryl substituents introduction in position 3 of the cycle.

For this purpose the scheme of interaction of $N^{1-}$ aryl/benzyl-3-hydrazinopyrazin-2-ones 4\{1-4\} with the corresponding carbonic acids $\mathbf{1}\{1-4\}$ has been suggested. However, low electrophility of the carboxylic group hinders the effective course of the reaction. Hence, for the carboxylic group activation we suggest to use carbonyldiimidazole (CDI) 2 in anhydrous DMFA in the molar ratio of 1:1 (Scheme).<smiles>[R2]C(=O)[O-]</smiles><smiles>[R2]C(=O)n1ccnc1</smiles><smiles>[R2]c1nnc2c(=O)n([R19])ccn12</smiles>

$\mathrm{R} 1=\mathrm{Ph}$; 4-FPh; 3,4-diFPh; 4-CIPhCH R2 = 3-CIPh; 3,4-diOMePh; 3,4-diOEtPh; 3-Pyr. 
3-Aryl/heteryl- $N^{7}$-aryl/benzyl[1,2,4]triazolo[4,3-a]pyrazin-8(7H)-ones

\begin{tabular}{|c|c|c|c|c|c|}
\hline $\begin{array}{l}\text { Compound } \\
\text { code }\end{array}$ & $\mathrm{R}$ & $\begin{array}{l}\text { Molecular } \\
\text { formula, } \\
\text { m.m. }\end{array}$ & $\begin{array}{c}\text { Yield, } \\
\%\end{array}$ & $\begin{array}{c}\mathrm{N}, \% \\
\text { Calc./ } \\
\text { found } \\
\end{array}$ & ${ }^{1-H}$ NMR spectral data $\delta$, ppm \\
\hline $\mathbf{5}\{1\}$ & $\begin{array}{c}\mathrm{R} 1=\mathrm{Ph} \\
\mathrm{R} 2=4-\mathrm{ClPh}\end{array}$ & $\begin{array}{l}\mathrm{C}_{17} \mathrm{H}_{11} \mathrm{ClN}_{4} \mathrm{O} \\
322.76\end{array}$ & 79 & $\begin{array}{c}17.36 / \\
17.39\end{array}$ & $\begin{array}{l}7.22(\mathrm{~d}, J=4.8,1 \mathrm{H}, \mathrm{H}-5) ; 7.38-7.54\left(\mathrm{~m}, 5 \mathrm{H}, \mathrm{H}-2^{\prime}, 3^{\prime}, 4^{\prime}, 5^{\prime}, 6^{\prime}\right) \\
7.57(\mathrm{~d}, J=4.8,1 \mathrm{H}, \mathrm{H}-6) ; 7.71\left(\mathrm{~d}, J=8.0,2 \mathrm{H}, \mathrm{H}-3^{\prime \prime}, 5^{\prime \prime}\right) ; \\
7.92\left(\mathrm{~d}, J=8.0,2 \mathrm{H}, \mathrm{H}-2^{\prime \prime}, 6^{\prime \prime}\right)\end{array}$ \\
\hline $\mathbf{5}\{2\}$ & $\begin{array}{l}\mathrm{R} 1=4-\mathrm{FPh} \\
\mathrm{R} 2=4-\mathrm{ClPh}\end{array}$ & $\begin{array}{c}\mathrm{C}_{17} \mathrm{H}_{10} \mathrm{ClFN}_{4} \mathrm{O} \\
340.75\end{array}$ & 80 & $\begin{array}{c}16.44 / \\
16.41\end{array}$ & $\begin{array}{l}7.18(\mathrm{~d}, J=4.9,1 \mathrm{H}, \mathrm{H}-5) ; 7.37\left(\mathrm{t}, J=8.2,2 \mathrm{H}, \mathrm{H}-3^{\prime}, 5^{\prime}\right) \\
7.51-7.59\left(\mathrm{~m}, 3 \mathrm{H}, \mathrm{H}-6,2^{\prime}, 6^{\prime}\right) ; 7.71\left(\mathrm{~d}, J=8.0,2 \mathrm{H}, \mathrm{H}-3^{\prime \prime}, 5^{\prime \prime}\right) ; \\
7.92\left(\mathrm{~d}, J=8.0,2 \mathrm{H}, \mathrm{H}-2^{\prime \prime}, 6^{\prime \prime}\right)\end{array}$ \\
\hline $5\{3\}$ & $\begin{array}{l}\mathrm{R} 1=4-\mathrm{OMePh} \\
\mathrm{R} 2=4-\mathrm{ClPh}\end{array}$ & $\begin{array}{c}\mathrm{C}_{18} \mathrm{H}_{13} \mathrm{ClN}_{4} \mathrm{O}_{2} \\
352.78\end{array}$ & 77 & $\begin{array}{l}15.88 / \\
15.92\end{array}$ & $\begin{array}{l}3.81\left(\mathrm{~s}, 3 \mathrm{H}, \mathrm{OCH} \mathrm{H}_{3}\right) ; 7.08-7.17\left(\mathrm{~m}, 3 \mathrm{H}, \mathrm{H}-5,3^{\prime}, 5^{\prime}\right) ; \\
7.38-7.54\left(\mathrm{~m}, 3 \mathrm{H}, \mathrm{H}-6,2^{\prime}, 6^{\prime}\right) ; 7.70\left(\mathrm{~d}, J=8.0,2 \mathrm{H}, \mathrm{H}-3^{\prime \prime}, 5^{\prime \prime}\right) ; \\
7.92\left(\mathrm{~d}, J=8.0,2 \mathrm{H}, \mathrm{H}-2^{\prime \prime}, 6^{\prime \prime}\right)\end{array}$ \\
\hline $\mathbf{5}\{4\}$ & $\begin{aligned} \mathrm{R} 1 & =4-\mathrm{ClPhCH}_{2} \\
\mathrm{R} 2 & =4-\mathrm{ClPh}\end{aligned}$ & $\begin{array}{c}\mathrm{C}_{18} \mathrm{H}_{12} \mathrm{Cl}_{2} \mathrm{~N}_{4} \mathrm{O} \\
371.23\end{array}$ & 78 & $\begin{array}{c}15.09 / \\
15.12\end{array}$ & $\begin{array}{l}5.11\left(\mathrm{~s}, 2 \mathrm{H}, \mathrm{NCH}_{2}\right) ; 7.28(\mathrm{~d}, J=4.9,1 \mathrm{H}, \mathrm{H}-5) ; \\
7.38-7.43\left(\mathrm{~m}, 4 \mathrm{H}, \mathrm{H}-2^{\prime}, 3^{\prime}, 5^{\prime}, 6^{\prime}\right) ; 7.59(\mathrm{~d}, J=4.9,1 \mathrm{H}, \mathrm{H}-6) ; \\
7.69\left(\mathrm{~d}, J=8.0,2 \mathrm{H}, \mathrm{H}-3^{\prime \prime}, 5^{\prime \prime}\right) ; 7.89\left(\mathrm{~d}, J=8.0,2 \mathrm{H}, \mathrm{H}-2^{2 \prime}, 6^{\prime \prime}\right)\end{array}$ \\
\hline $\mathbf{5}\{5\}$ & $\begin{aligned} & \mathrm{R} 1=\mathrm{Ph} \\
& \mathrm{R} 2= 3,4-\mathrm{diOMePh}\end{aligned}$ & $\begin{array}{c}\mathrm{C}_{19} \mathrm{H}_{16} \mathrm{~N}_{4} \mathrm{O}_{3} \\
348.36\end{array}$ & 70 & $\begin{array}{c}15.09 / \\
15.12\end{array}$ & $\begin{array}{l}3.87(\mathrm{~s}, 6 \mathrm{H}, 2 \mathrm{OCH}) ; 7.14-7.23\left(\mathrm{~m}, 2 \mathrm{H}, \mathrm{H}-5,5^{\prime \prime}\right) ; \\
7.34-7.64\left(\mathrm{~m}, 8 \mathrm{H}, \mathrm{H}-6,2^{\prime} 3^{\prime} \mathrm{4}^{\prime}, 5^{\prime}, 6^{\prime}, 2^{\prime \prime}, 6^{\prime \prime}\right)\end{array}$ \\
\hline $5\{6\}$ & $\begin{array}{c}\mathrm{R} 1=4-\mathrm{FPh} \\
\mathrm{R} 2=3,4-\mathrm{diOMePh}\end{array}$ & $\begin{array}{c}\mathrm{C}_{19} \mathrm{H}_{15} \mathrm{FN}_{4} \mathrm{O}_{3} \\
366.35\end{array}$ & 68 & $\begin{array}{l}15.29 / \\
15.27 \\
\end{array}$ & $\begin{array}{l}\left.3.87(\mathrm{~s}, 6 \mathrm{H}, 2 \mathrm{OCH})_{3}\right) ; 7.14-7.23\left(\mathrm{~m}, 2 \mathrm{H}, \mathrm{H}-5,5^{\prime \prime}\right) ; \\
\left.\text { 7.34-7.46 (m, } 4 \mathrm{H}, \mathrm{H}-3^{\prime}, 5^{\prime}, 2^{\prime \prime}, 6^{\prime \prime}\right) ; 7.55-7.64\left(\mathrm{~m}, 3 \mathrm{H}, \mathrm{H}-6,2^{\prime} \mathrm{6}^{\prime}\right)\end{array}$ \\
\hline $5\{7\}$ & $\begin{aligned} \mathrm{R} 1 & =4-\mathrm{OMePh} \\
\mathrm{R} 2 & =3,4-\mathrm{diOMePh}\end{aligned}$ & $\begin{array}{c}\mathrm{C}_{20} \mathrm{H}_{18} \mathrm{~N}_{4} \mathrm{O}_{4} \\
378.39\end{array}$ & 69 & $\begin{array}{l}14.81 / \\
14.81\end{array}$ & $\begin{array}{l}3.81\left(\mathrm{~s}, 3 \mathrm{H}, 4^{\prime}-\mathrm{OCH}_{3}\right) ; 3.87\left(\mathrm{~s}, 6 \mathrm{H}, 3^{\prime \prime}, 4^{\prime \prime} \mathrm{OCH}_{3}\right) ; \\
\left.\text { 7.06-7.14 (m, 3H, H-3,', } 5^{\prime}, 5^{\prime \prime}\right) ; 7.22(\mathrm{~d}, J=4.8,1 \mathrm{H}, \mathrm{H}-5) ; \\
7.38-7.44\left(\mathrm{~m}, 4 \mathrm{H}, \mathrm{H}-2^{\prime}, 6^{\prime}, 2^{\prime \prime}, 6^{\prime \prime}\right) ; 7.58(\mathrm{~d}, J=4.8,1 \mathrm{H}, \mathrm{H}-6)\end{array}$ \\
\hline $\mathbf{5}\{8\}$ & $\begin{aligned} \mathrm{R} 1 & =4-\mathrm{ClPhCH}_{2} \\
\mathrm{R} 2 & =3,4-\mathrm{diOMePh}\end{aligned}$ & $\begin{array}{c}\mathrm{C}_{20} \mathrm{H}_{17} \mathrm{ClN}_{4} \mathrm{O}_{3} \\
396.84\end{array}$ & 72 & $\begin{array}{c}14.12 / \\
14.16\end{array}$ & $\begin{array}{l}3.84\left(\mathrm{~s}, 6 \mathrm{H}, 2 \mathrm{OCH} \mathrm{H}_{3}\right) ; 5.11\left(\mathrm{~s}, 2 \mathrm{H}, \mathrm{NCH}_{2}\right) ; 7.17(\mathrm{~d}, J=7.8 \\
\left.1 \mathrm{H}, \mathrm{H}^{\prime \prime} 5^{\prime \prime}\right) ; 7.24(\mathrm{~d}, J=4.9,1 \mathrm{H}, \mathrm{H}-5) ; 7.34-7.43(\mathrm{~m}, 6 \mathrm{H}, \\
\left.\mathrm{H}-2^{\prime}, 3^{\prime}, 5^{\prime}, 6^{\prime}, 2^{\prime \prime}, 6^{\prime \prime}\right) ; 7.59(\mathrm{~d}, J=4.9,1 \mathrm{H}, \mathrm{H}-6)\end{array}$ \\
\hline $\mathbf{5}\{9\}$ & $\begin{array}{c}\mathrm{R} 1=\mathrm{Ph} \\
\mathrm{R} 2=3,4 \mathrm{diOEtPhCH}\end{array}$ & $\begin{array}{c}\mathrm{C}_{22} \mathrm{H}_{22} \mathrm{~N}_{4} \mathrm{O}_{3} \\
390.45\end{array}$ & 75 & $\begin{array}{c}14.35 / \\
14.32\end{array}$ & $\begin{array}{l}1.35\left(\mathrm{t}, J=7.6,6 \mathrm{H}, 2 \mathrm{CH}_{3}\right) ; 4.08\left(\mathrm{qr}, J=7.6,4 \mathrm{H}, 2 \mathrm{OCH}_{2}\right) ; \\
4.33\left(\mathrm{~s}, 2 \mathrm{H}, \mathrm{CH} \mathrm{H}_{2}\right) ; 6.77\left(\mathrm{~d}, J=7.8,1 \mathrm{H}, \mathrm{H}-5^{\prime \prime}\right) ; 6.88(\mathrm{~d}, J= \\
\left.7.8,1 \mathrm{H}, \mathrm{H}-6^{\prime \prime}\right) ; 6.95\left(\mathrm{~s}, 1 \mathrm{H}, \mathrm{H}-2^{\prime \prime}\right) ; 7.15(\mathrm{~d}, J=4.8,1 \mathrm{H}, \mathrm{H}-5) ; \\
7.38-7.54\left(\mathrm{~m}, 6 \mathrm{H}, \mathrm{H}-6,2^{\prime}, 3^{\prime} 4^{\prime} \mathrm{\prime}^{\prime} 5^{\prime}, 6^{\prime}\right)\end{array}$ \\
\hline $\mathbf{5}\{10\}$ & $\begin{array}{c}\mathrm{R} 1=4-\mathrm{FPh} \\
\mathrm{R} 2=3,4 \mathrm{diOEtPhCH}\end{array}$ & $\begin{array}{l}\mathrm{C}_{22} \mathrm{H}_{21} \mathrm{FN}_{4} \mathrm{O}_{3} \\
408.44\end{array}$ & 77 & $\begin{array}{c}13.72 / \\
13.75\end{array}$ & $\begin{array}{l}\left.1.35\left(\mathrm{t}, J=7.6,6 \mathrm{H}, 2 \mathrm{CH}_{3}\right) ; 4.08(\mathrm{qr}, J=7.6,4 \mathrm{H}, 2 \mathrm{OCH})_{2}\right) \\
4.37\left(\mathrm{~s}, 2 \mathrm{H}, \mathrm{CH}_{2}\right) ; 6.77\left(\mathrm{~d}, J=7.8,1 \mathrm{H}, \mathrm{H}-5^{\prime \prime}\right) ; 6.88(\mathrm{~d}, J= \\
\left.7.8,1 \mathrm{H}, \mathrm{H}-6^{\prime \prime}\right) ; 6.98\left(\mathrm{~s}, 1 \mathrm{H}, \mathrm{H}-2^{\prime \prime}\right) ; 7.18(\mathrm{~d}, J=4.9,1 \mathrm{H}, \mathrm{H}-5) ; \\
7.37\left(\mathrm{t}, J=8.2,2 \mathrm{H}, \mathrm{H}-3^{\prime}, 5^{\prime}\right) ; 7.51-7.59\left(\mathrm{~m}, 3 \mathrm{H}, \mathrm{H}-6,2^{\prime}, 6^{\prime}\right)\end{array}$ \\
\hline $\mathbf{5}\{11\}$ & $\begin{array}{c}\mathrm{R} 1=4-\mathrm{OMePh} \\
\mathrm{R} 2=3,4 \mathrm{diOEtPhCH}\end{array}$ & $\begin{array}{c}\mathrm{C}_{23} \mathrm{H}_{24} \mathrm{~N}_{4} \mathrm{O}_{4} \\
420.47\end{array}$ & 73 & $\begin{array}{c}13.32 / \\
13.31\end{array}$ & $\begin{array}{l}1.35\left(\mathrm{t}, J=7.6,6 \mathrm{H}, 2 \mathrm{CH}_{3}\right) ; 3.81\left(\mathrm{~s}, 3 \mathrm{H}, \mathrm{OCH}_{3}\right) ; 4.08(\mathrm{qr}, \\
\left.J=7.6,4 \mathrm{H}, 2 \mathrm{OCH} \mathrm{H}_{2}\right) ; 4.35\left(\mathrm{~s}, 2 \mathrm{H}, \mathrm{CH}_{2}\right) ; 6.77(\mathrm{~d}, J=7.8,1 \mathrm{H}, \\
\left.\mathrm{H}-5^{\prime \prime}\right) ; 6.88\left(\mathrm{~d}, J=7.8,1 \mathrm{H}, \mathrm{H}-6^{\prime \prime}\right) ; 6.98\left(\mathrm{~s}, 1 \mathrm{H}, \mathrm{H}-2^{\prime \prime}\right) ; 7.08 \\
\left(\mathrm{~d}, J=7.8,2 \mathrm{H}, \mathrm{H}-3^{\prime}, 5^{\prime}\right) ; 7.20(\mathrm{~d}, J=4.8,1 \mathrm{H}, \mathrm{H}-5) ; 7.33(\mathrm{~d}, \\
\left.J=7.8,2 \mathrm{H}, \mathrm{H}-2^{\prime} 6^{\prime}\right) ; 7.50(\mathrm{~d}, J=4.8,1 \mathrm{H}, \mathrm{H}-6)\end{array}$ \\
\hline $\mathbf{5}\{12\}$ & $\begin{array}{r}\mathrm{R} 1=4-\mathrm{ClPhCH}_{2} \\
\mathrm{R} 2=3,4 \mathrm{diOEtPhCH}\end{array}$ & $\begin{array}{c}\mathrm{C}_{23} \mathrm{H}_{23} \mathrm{ClN}_{4} \mathrm{O}_{3} \\
438.92\end{array}$ & 65 & $\begin{array}{c}12.76 / \\
12.79\end{array}$ & $\begin{array}{l}\left.1.35\left(\mathrm{t}, J=7.6,6 \mathrm{H}, 2 \mathrm{CH}_{3}\right) ; 4.08\left(\mathrm{qr}_{1} J=7.6,4 \mathrm{H}, 2 \mathrm{OCH}\right)_{2}\right) ; \\
4.33\left(\mathrm{~s}, 2 \mathrm{H}, \mathrm{CH}_{2}\right) ; 5.07\left(\mathrm{~s}, 2 \mathrm{H}, \mathrm{NCH} \mathrm{H}_{2}\right) ; 6.77(\mathrm{~d}, J=7.8,1 \mathrm{H}, \\
\left.\mathrm{H}-5^{\prime \prime}\right) ; 6.88\left(\mathrm{~d}, J=7.8,1 \mathrm{H}, \mathrm{H}-6^{\prime \prime}\right) ; 6.95\left(\mathrm{~s}, 1 \mathrm{H}, \mathrm{H}-2^{\prime \prime}\right) ; 7.22 \\
(\mathrm{~d}, J=4.9,1 \mathrm{H}, \mathrm{H}-5) ; 7.33-7.40\left(\mathrm{~m}, 4 \mathrm{H}, \mathrm{H}-2^{\prime}, 3^{\prime}, 5^{\prime}, 6^{\prime}\right) ; 7.55(\mathrm{~d}, \\
J=4.9,1 \mathrm{H}, \mathrm{H}-6)\end{array}$ \\
\hline $\mathbf{5}\{13\}$ & $\begin{array}{c}\mathrm{R} 1=\mathrm{Ph} \\
\mathrm{R} 2=3-\mathrm{Pyr}\end{array}$ & $\begin{array}{c}\mathrm{C}_{16} \mathrm{H}_{11} \mathrm{~N}_{5} \mathrm{O} \\
289.30\end{array}$ & 81 & $\begin{array}{l}24.21 / \\
24.22\end{array}$ & 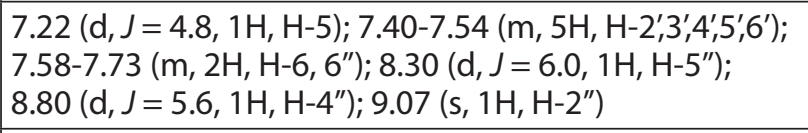 \\
\hline $\mathbf{5}\{14\}$ & $\begin{array}{l}\mathrm{R} 1=4-\mathrm{FPh} \\
\mathrm{R} 2=3-\mathrm{Pyr}\end{array}$ & $\begin{array}{c}\mathrm{C}_{16} \mathrm{H}_{10} \mathrm{FN}_{5} \mathrm{O} \\
307.29\end{array}$ & 82 & $\begin{array}{l}22.79 / \\
22.78\end{array}$ & $\begin{array}{l}7.17(\mathrm{~d}, J=4.9,1 \mathrm{H}, \mathrm{H}-5) ; 7.37\left(\mathrm{t}, J=8.2,2 \mathrm{H}, \mathrm{H}-3^{\prime}, 5^{\prime}\right) ; 7.45- \\
7.55\left(\mathrm{~m}, 2 \mathrm{H}, \mathrm{H}-2^{\prime}, 6^{\prime}\right) ; 7.58-7.70\left(\mathrm{~m}, 2 \mathrm{H}, \mathrm{H}-6,6^{\prime \prime}\right) ; 8.30(\mathrm{~d}, \\
\left.J=6.0,1 \mathrm{H}, \mathrm{H}-5^{\prime \prime}\right) ; 8.80\left(\mathrm{~d}, J=5.6,1 \mathrm{H}, \mathrm{H}-4^{\prime \prime}\right) ; 9.07\left(\mathrm{~s}, 1 \mathrm{H}, \mathrm{H}-2^{\prime \prime}\right)\end{array}$ \\
\hline $\mathbf{5}\{15\}$ & $\begin{array}{c}\mathrm{R} 1=4-\mathrm{OMePh} \\
\mathrm{R} 2=3-\mathrm{Pyr}\end{array}$ & $\begin{array}{c}\mathrm{C}_{17} \mathrm{H}_{13} \mathrm{~N}_{5} \mathrm{O}_{2} \\
319.33\end{array}$ & 79 & $\begin{array}{c}21.93 / \\
21.94\end{array}$ & $\begin{array}{l}\left.3.81(\mathrm{~s}, 3 \mathrm{H}, \mathrm{OCH})_{3}\right) ; .08\left(\mathrm{~d}, J=7.8,2 \mathrm{H}, \mathrm{H}-3^{\prime}, 5^{\prime}\right) ; 7.17(\mathrm{~d}, \\
J=4.8,1 \mathrm{H}, \mathrm{H}-5) ; 7.39\left(\mathrm{~d}, J=7.8,2 \mathrm{H}, \mathrm{H}-2^{\prime}, 6^{\prime}\right) ; 7.58-7.70 \\
\left(\mathrm{~m}, 2 \mathrm{H}, \mathrm{H}-6,6^{\prime \prime}\right) ; 8.30\left(\mathrm{~d}, J=6.0,1 \mathrm{H}, \mathrm{H}-5^{\prime \prime}\right) ; 8.80(\mathrm{~d}, J= \\
\left.5.6,1 \mathrm{H}, \mathrm{H}-4^{\prime \prime}\right) ; 9.07\left(\mathrm{~s}, 1 \mathrm{H}, \mathrm{H}-2^{\prime \prime}\right)\end{array}$ \\
\hline $\mathbf{5}\{16\}$ & $\begin{array}{c}\mathrm{R} 1=4-\mathrm{ClPhCH}_{2} \\
\mathrm{R} 2=3-\mathrm{Pyr}\end{array}$ & $\begin{array}{c}\mathrm{C}_{17} \mathrm{H}_{12} \mathrm{ClN}_{5} \mathrm{O} \\
337.77\end{array}$ & 83 & $\begin{array}{c}20.73 / \\
20.73\end{array}$ & $\begin{array}{l}5.11\left(\mathrm{~s}, 2 \mathrm{H}, \mathrm{NCH}_{2}\right) ; 7.22(\mathrm{~d}, J=4.9,1 \mathrm{H}, \mathrm{H}-5) ; 7.33-7.40(\mathrm{~m}, \\
\left.4 \mathrm{H}, \mathrm{H}-2^{\prime}, 3^{\prime}, 5^{\prime}, 6^{\prime}\right) ; 7.53-7.65\left(\mathrm{~m}, 2 \mathrm{H}, \mathrm{H}-6,66^{\prime \prime}\right) ; 8.30(\mathrm{~d}, J=6.0, \\
\left.1 \mathrm{H}, \mathrm{H}-5^{\prime \prime}\right) ; 8.80\left(\mathrm{~d}, J=5.6,1 \mathrm{H}, \mathrm{H}-4^{\prime \prime}\right) ; 9.07\left(\mathrm{~s}, 1 \mathrm{H}, \mathrm{H}-2^{\prime \prime}\right)\end{array}$ \\
\hline
\end{tabular}


Thus, imidazolide of the corresponding acid $3\{1-4\}$ is formed; it is very suitable reagent for the synthesis of intermediate hydrazides, which with further heating form the target products that precipitate. Possible impurities (starting acid or its imidazolide) are more soluble than the final product and stay in solution when precipitating.

The use of acid chlorides is less appropriate for the target products synthesis because of the presence of the thionyl chloride excess, and it can lead to formation of by-products impurities.

It should be mentioned that attempts to conduct the synthesis of compounds $\mathbf{5}$ by interaction of 3-chloropyrazin-2-ones and the corresponding acid hydrazides have failed since starting chlorides are easily hydrolyzed by traces of water in solvents or even by air moisture.

The structures of 3-substituted [1,2,4]triazolo[4,3- $a]$ pyrazin-8 $(7 H)$-ones $\mathbf{5}\{1-16\}$ obtained have been confirmed by ${ }^{1} \mathrm{H}$ NMR spectroscopy data (Table). Formation of $[1,2,4]$ triazolo $[4,3-a]$ pyrazin- $8(7 H)$-one condensed system is in good correlation with spectral data. Thus, in addition to the signals of substituents in positions 3 and 7 of the heterocycle in ${ }^{1} \mathrm{H}$ NMR spectra, only signals of $\mathrm{H}-5$ and $\mathrm{H}-6$ protons of the pyrazinone fragment are present as doublets at $\delta$ 7.15-7.28 ppm and $\delta$ 7.50-7.59 ppm, respectively.

\section{Experimental Part}

General procedure for the synthesis of 3-aryl/ heteryl- $N^{7}$-aryl/benzyl $[1,2,4]$ triazolo $[4,3-a]$ pyrazin-8 $(7 \mathrm{H})$-ones $5\{1-16\}$. Dissolve the mixture of $15 \mathrm{mmol}$ of the corresponding acid $\mathbf{1}\{1-4\}$ and $2.43 \mathrm{~g}$ of ( $15 \mathrm{mmol}$ ) of carbonyldiimidazole 2 in $10 \mathrm{ml}$ of anhydrous DMFA. Heat the solution at $100^{\circ} \mathrm{C}$ for 1 hour, then add $10 \mathrm{mmol}$ of $N^{1}$-aryl/benzyl-3-hydrazinopyrazin-2-one 4\{1-4\}. Reflux the reaction mixture for 24 hours. After cooling dilute it with $100 \mathrm{ml}$ of water. Next day filter the precipitate formed, wash with $200 \mathrm{ml}$ of $i$-propanol and recrystallize from the mixture with $10 \mathrm{ml}$ of DMFA and $20 \mathrm{ml}$ of $i$-propanol.

\section{Conclusions}

The method suggested allows to obtain a great variety of target products - 3-substituted $[1,2,4]$ triazolo[4,3-a]pyrazin-8(7H)-ones using practically any acid for transformation.

\section{References}

1. Reinhard Sarges, Harry R. Howard, Roland G. Browne, Lorraine A. Lebel, Patricia A. Seymour, B. Kenneth Koe. J. of Medicinal Chemistry, 1990, Vol. 33, No.8, pp.2240-2254.

2. Mignani S., Stutzmann J.-M., Vuilhorgne M. Trends in Heterocyclic Chemistry, 2002, Vol. 8, pp.49-60.

3. Orechovich V.N. Institute of Biomedical Chemistry. PASS: http://www.pharmaexpert.ru/PASSOnline/.

4. Kovalenko S.S., Kulikovska K.Yu., Drushlyak O.G., Zhuravel I.O., Kovalenko S.M., Chernykh V.P. Chem. of Heterocyclic Compounds, 2014, Impress.

5. Schneller S.W., May J.L. J. Heterocyclic Chem, 1978, Vol. 15, pp.987-992.

Надійшла до редакції 21.04.2014 р. 